• 研究报告・

\title{
植原体tuf基因与其上游部分基因结构和相关 基因启动子保守区域特征及活性分析
}

\author{
于少帅 林彩丽 王圣洁 张文金国国忠 ${ }^{*}$
}

(中国林业科学研究院森林生态环境与保护研究所, 国家林业局森林保护学重点实验室, 北京 100091)

\begin{abstract}
摘要: 植原体寄主种类多, 危害范围广, 开展其遗传多样性、关键基因调控等方面研究有助于提高该病害综合防 治水平。通过长片段PCR引物扩增我国PaWB-sdyz、PaWB-fjfz和LY-fjya1植原体株系tuf基因及其上游 6 个基因的片 段, 进行植原体基因启动子保守区域序列特征和多位点序列分析。利用启动子探针载体pSUPV4检测植原体tuf基 因上游序列的启动子活性。扩增获得PaWB-sdyz、PaWB-fjfz、LY-fjya1株系tuf基因上游12,745-12,748 bp序列，比 较分析发现PaWB-sdyz、PaWB-fjfz、LY-fjya1、OY-M、AYWB、PAa、SLY、AT植原体株系tuf与其上游6个基因 的结构顺序皆为 5'-rplL-rpoB-rpoC-rps12-rps7-fusA-tuf-3'。推测出可能的植原体启动子保守区域模式序列: $\mathrm{T}_{90} \mathrm{~T}_{100} \mathrm{G}_{92} \mathrm{~T}_{75} \mathrm{G}_{67} \mathrm{~A}_{85}$ (-35区); $\mathrm{T}_{90} \mathrm{~A}_{96} \mathrm{~T}_{92} \mathrm{~A}_{98} \mathrm{~T}_{73} \mathrm{~T}_{90}$ (-10区)。基于 8 个植原体株系的rplL-tuf核苷酸序列编码基因、非编 码序列、氨基酸序列的多位点序列分析可将不同植原体株系以较高的支持率清晰地区分，不同植原体株系r r lL-tuf 核苷酸非编码区变异水平更高。16SrI组植原体tuf基因上游序列存在3种变异类型，其代表株系PaWB-fjfz、LY-fjya1 tuf基因上游130 bp片段和CWB-hnsy1 tuf基因上游129 bp片段皆具有启动子活性。
\end{abstract}

关键词：植原体; 启动子; 基因结构; 遗传多样性; 多位点序列分析

\section{Structures of the tuf gene and its upstream part genes and characteristic analysis of conserved regions and activity from related gene promoters of a phytoplasma}

\author{
Shaoshuai Yu, Caili Lin, Shengjie Wang, Wenxin Zhang, Guozhong Tian * \\ Key Laboratory of Forest Protection of State Forestry Administration, Research Institute of Forest Ecology, Environment \\ and Protection, Chinese Academy of Forestry, Beijing 100091
}

\begin{abstract}
Studies on the genetic diversity, key gene regulation and control of phytoplasma, which cause many diseases with various host plants and have a wide geographical distribution, will be conducive to facilitating integrated disease control. The large DNA fragments including tuf gene sequences and upstream six genes from PaWB-sdyz, PaWB-fjfz and LY-fjya1 strains were amplified using long fragment PCR primers. Sequence characteristic of conserved regions of the phytoplasma gene promoter and MLSA were performed. Upstream sequences adjoining the tuf gene was recombined with promoter-probe vector pSUPV4 to analyze their promoter activity. The sequences, 12,745-12,748 bp in length, of upstream tuf genes were amplified from the three strains. Comparative analysis showed that the gene structure order of the tuf gene and its upstream six gene sequences of PaWB-sdyz, PaWB-fjfz, LY-fjya1, OY-M, AYWB, PAa, SLY, AT phytoplasma strains were identical in the arrangement of 5'-rplL-rpoB-rpoC-rps12-rps7-fusA-tuf-3'. The potential sequence pattern of conserved region of the phytoplasma promoter was deduced: $\mathrm{T}_{90} \mathrm{~T}_{100} \mathrm{G}_{92} \mathrm{~T}_{75} \mathrm{G}_{67} \mathrm{~A}_{85}$ ( -35 region); $\mathrm{T}_{90} \mathrm{~A}_{96} \mathrm{~T}_{92} \mathrm{~A}_{98} \mathrm{~T}_{73} \mathrm{~T}_{90}$ (-10 region). The different phytoplasma strains were clearly divided with comparatively high bootstrap values based on MLSA of coding genes, non-coding sequences, and deduced amino acid sequences of rplL-tuf nucleotide sequences. Genetic variation was comparatively high in the non-coding nucleotide sequences. A 130-bp upstream sequence of the tuf gene in PaWB-fjfz, LY-fjya1 strains and a
\end{abstract}

收稿日期: 2018-01-16; 接受日期: 2018-03-26

基金项目: 国家自然科学基金(31370644)

* 通讯作者 Author for correspondence. E-mail: tian3691@163.com 
129-bp upstream sequence of the tuf gene in CWB-hnsy1 strain, and three representative strains of three variation types of upstream sequences adjoining the tuf gene from 16SrI group, were tested for promoter activity.

Key words: phytoplasma; promoter; gene structure; genetic diversity; multilocus sequence analysis

植原体(phytoplasma) 是一类寄生于植物韧皮 部、无细胞壁的植物致病细菌, 其植物寄主的种类 多、地理分布范围广, 造成的危害严重。植原体基 因组大小在530-1,350 kb之间, 目前完成全基因组 测序的植原体株系有 5 个, 分别为 $16 \mathrm{SrI}$ 组的洋苞黄 化植原体(onion yellows phytoplasma, OY-M) (GenBank 登录号AP006628) 和翠菊黄化丛枝植原体 (aster yellows witches'-broom phytoplasma, AYWB) (CP000061), 16SrXII组的澳大利亚葡萄黄化植原体 (Candidatus phytoplasma australiense, PAa) (AM422018)和草莓致死黄化植原体(strawberry lethal yellows phytoplasma, SLY) (CP002548), 还有16SrX组 的苹果簇生植原体 (Ca. P. mali, AT) (CU469464) (Oshima et al, 2004; Bai et al, 2006; Tran-Nguyen et al, 2008; Kube et al, 2008; Andersen et al, 2013)。不 同植原体株系寄主种类、引起病害症状、相关病害 地理分布等都有差异。从基因组水平分析发现不同 植原体株系基因组大小、染色体形态、G + C含量、 质粒数量、开放阅读框 (open reading frame, ORF) 数 量、基因结构和功能等均有明显差异(于少帅等, 2016a)。

已知原核生物操纵子的基因结构和其调控序 列的变化都会影响到操纵子的表达。研究发现大肠 杆菌rps12、rps7、fusA、tuf共同组成了一个str操纵 子, rps12前和tuf后分别存在启动子结构和转录终止 结构(Post \& Nomura, 1980; 朱玉贤和李毅, 2002)。 大肠杆菌中rpS10基因在 $s t r$ 操纵子下游 $15 \mathrm{~kb}$ 处, 与 核糖体蛋白编码基因 rpl3、rpl4等组成 $S 10$ 操纵子, 在S10操纵子3, 端发现转录终止结构(Post \& Nomura, 1980; Zurawski \& Zurawski, 1985)。Sanangelantoni等(1993)研究发现甲烷叶菌(Methanococcus vannielii)的核糖体蛋白编码基因 rpS10与tuf基因连 在一起与 $s t r$ 操纵子一起转录。 $s p c$ 操纵子和 $S 10$ 操纵 子在大肠杆菌基因组中均有发现(Post \& Nomura, 1980; Zurawski \& Zurawski, 1985)。Miyata等(2002a) 在植原体 $S 10$ 操纵子和 $s p c$ 操纵子之间没有找到与转
录起始或终止相关的序列, 而在 $S 10$ 操纵子上游邻 接序列发现了可能的启动子结构, 推测 $S 10$ 操纵子 和 $s p c$ 操纵子在植原体中是一个转录单位, 构成 S10-spc操纵子。Miyata等(2002b)推断洋苟黄化植原 体OY的 str操纵子的结构与大肠杆菌的 $s t r$ 操纵子结 构排布一致, 为5'-rps12-rps7-fusA-tuf-3'。与支原体 相比, 洋葱黄化植原体OY的 str操纵子基因结构排 布与芽孢杆菌更接近(Miyata et al, 2002b), 但研究 未通过实验验证预测启动子的功能活性。

启动子(promoter)是RNA聚合酶结合到模板完 成转录起始步骤所需的DNA序列, 转录起始是基因 表达的关键阶段(朱玉贤和李毅, 2002; 李明刚等, 2004)。病原菌基因结构变化和启动子序列的有无及 变异会改变相关基因的表达及代谢效率, 并对病原 菌与其寄主的互作关系产生重要影响(Ishii et al, 2009; Du et al, 2014; Zhang et al, 2014)。Du等(2014) 发现猪乳糖酶基因启动子和增强子中存在较丰富 的单核苷酸多态性, 这些调控序列的碱基变异导致 基因表达水平发生显著差异。Zhang等(2014)研究发 现, 人类的 $\mathrm{T}-\mathrm{box}$ 转录因子基因 TBX1调控序列碱基 发生变异将改变 TBX1基因的表达水平, 而 TBX1基 因表达水平的改变与人类疾病腹股沟疝的产生有 一定关系。Ishii等(2009)对洋葱黄化植原体非虫传 株系 OY-NIM质粒EcOYM和野生株系 OY-M质粒 EcOYNIM 上编码膜蛋白基因 orf3 的启动子 ORF3-pro1和ORF3-pro2 的保守结构进行了分析, 并与 $16 \mathrm{~S} \mathrm{rRNA}$ 和 $A m p$ 基因的启动子结构进行了软 件预测与比较分析, 发现OY-NIM株系ORF3-pro2 启动子缺失, 从而推断膜蛋白ORF3 在洋苟黄化植 原体与昆虫互作过程中起到重要作用, 但研究尚缺 乏启动子功能验证实验的直接证据。

我们对我国16SrI组的泡桐丛枝植原体PaWBsdyz株系tuf基因及其邻接上游序列分析发现其上游 序列存在明显的启动子结构, 经PSUPV4启动子探 针检测具有启动子活性, 由此推测泡桐从枝植原体 的 tuf基因可能单独表达(于少帅等, 2016b)。已有 
研究发现：除 PaWB-sdyz株系tuf基因启动子外, 16SrI组植原体fusA-tuf基因间区序列还存在其他3种 变异类型, 均预测有完整的启动子结构(于少帅等, 2016b), 但启动子活性未做验证。而启动子序列的 变异对启动子活性的有无影响较大(Ishii et al, 2009; Du et al, 2014; Zhang et al, 2014), 因此, 本研究对 其他 3 种变异类型的启动子活性进行了分析。在此 基础上, 本研究进一步通过长片段PCR扩增我国泡 桐从枝植原体山东充州株系PaWB-sdyz、福建福州 株系PaWB-fjfz和莴苣黄化植原体福建永安株系 LY-fjya1基因tuf及其上游6个基因 $r p l L 、 r p o B 、 r p o C$ 、 $r p s 12 、 r p s 7 、 f u s A$ 序列，结合全基因测序已完成的 5 个植原体株系和非固醇甾原体(Acholeplasma laidlawii) PG-8A株系的tuf及其上游基因序列进行比较 分析，试图揭示不同植原体tuf基因与其上游部分基 因结构排布及序列间的差异，阐明植原体基因启动 子的保守区域序列特征，从基因水平上认识不同植 原体株系间的差异, 深入揭示植原体与植物寄主和 应性等问题，从而达到防治相关病害的目的。

\section{材料与方法}

\section{1 材料}

供试样品: 泡桐丛枝病植原体PaWB-sdyz和 PaWB-fjfz来自实验室组培苗和福建福州, 莴苣黄 化植原体LY-fjya1和苦棟丛枝植原体CWB-hnsy1采 自福建永安和海南三亚，样品采于2013-2014年间。 每个采样地采集植物健康枝叶作为对照。

试剂及仪器: 限制性内切酶Hind III和BamH I、 T4 DNA连接酶、大肠杆菌感受态细胞DH5 $\alpha$ 、高保 真LA Taq DNA聚合酶等购自日本TaKaRa公司，2× PCR Mix购自北京博迈德公司, 植物基因组提取试 剂盒、Marker试剂、SYBR Green I染料购自北京艾 德莱公司, pSUPV4质粒由四川大学王海燕副教授 提供, 其他试剂均为国产分析纯。LongGene A200 型基因扩增仪, 北京市六一仪器厂DYY-6C型电泳 仪。引物由北京赛百盛公司合成, DNA测序由生工 生物工程(上海)股份有限公司完成。

\section{2 方法}

\subsection{1 植原体总DNA的提取和检测}

通过CTAB法提取植物中植原体总DNA, 参照 艾德莱植物基因组提取试剂盒提供方法。用引物对

R16mF2/R16mR1 (Lee et al, 1993) PCR扩增测序检 测植原体。PCR反应设阳性对照(实验室组培的感染 植原体植物)和阴性对照(健康植物)。

\subsection{2 植原体tuf及其上游部分基因长片段扩增} 根据OY-M基因组序列设计4对引物，采用高保 真LA Taq DNA聚合酶扩增PaWB-sdyz、PaWB-fjfz、 LY-fjya1植原体株系tuf基因及其上游6个基因及间 区约13 kb的序列, 7 个基因的结构顺序为 $5^{\prime}-r p l L-$ rpoB-rpoC-rps12-rps7-fusA-tuf -3'。编码蛋白分别为 rplL: 核糖体蛋白L7/L12 (ribosomal protein L7/L12); rpoB: RNA聚合酶beta亚基(DNA-directed RNA polymerase beta subunit); rpoC: RNA聚合酶beta'亚基 (DNA-directed RNA polymerase beta' subunit); rps12: 核糖体蛋白S12 (ribosomal protein S12); rps7: 核糖 体蛋白S7 (ribosomal protein S7); fusA: 蛋白延伸因 子EF-G (translation elongation factor G); tuf: 蛋白延 伸因子EF-Tu (translation elongation factor Tu)。

PCR反应体系为 $50 \mu \mathrm{L}$, 包括 $2.0 \mu \mathrm{L}$ DNA提取 物, $0.5 \mu \mathrm{L}$ 上游引物和下游引物 $(10 \mu \mathrm{M}), 0.5 \mu \mathrm{L} \mathrm{LA}$ Taq DNA聚合酶 $(5 \mathrm{U} / \mu \mathrm{L}), 5 \mu \mathrm{L} 10 \times$ LA Taq DNA聚 合酶buffer II ( $\mathrm{Mg}^{2+}$ Plus), $8 \mu \mathrm{L}$ dNTP (2.5 mM each), 用 $\mathrm{ddH}_{2} \mathrm{O}$ 补至 $50 \mu \mathrm{L}$ 。引物信息如表1所示。

\subsection{3 植原体启动子保守结构特征序列}

利用DNAStar和DNAMAN 5.0软件拼接所得序 列，并将所得序列与植原体参考株系 OY-M、 AYWB、PAa、SLY、AT和非固醇甾原体PG-8A相 关DNA序列进行多重序列比对, 通过 Softberry (http://linux1.softberry.com/berry.phtml) 在线分析工 具并参考相关资料(朱玉贤和李毅，2002; Turner, 2010)推测不同植原体株系tuf基因及上游基因间区 序列可能存在的启动子及其结构。对植原体基因启 动子保守区域不同位置核苷酸种类及出现频率进 行统计分析，推测植原体基因启动子保守区域可能 的序列模式。

\subsubsection{MLSA分析}

利用MEGA7.0软件的邻接法构建系统发育树 (Kumar et al, 2016)。自展值设为1,000, 用以评估软 件生成的系统进化树的稳定性和支持率 (Felsenstein, 1985)。OY-M、AYWB、PAa、SLY、AT和非 固醇甾原体PG-8A相关DNA序列作为构建系统发 育树的外类群。采用 $r p l L 、 r p o B 、 r p o C 、 r p s 12 、 r p s 7$ 、 fusA、tuf共7个基因和其对应的氨基酸序列 RplL、 
表1 长片段DNA扩增引物信息

Table 1 Information of the primers used to amplify the large DNA fragments

\begin{tabular}{|c|c|c|c|c|c|}
\hline 引物 Primer & 序列 Sequence (5’-3’) & 长度 Length (nt) & $\mathrm{T}_{\mathrm{m}}\left({ }^{\circ} \mathrm{C}\right)$ & $\mathrm{G}+\mathrm{C}(\%)$ & 扩增长度 Length amplified (bp) \\
\hline op1 & 5'-GATTGACATGGCTAAGTTAACG-3' & 22 & 54.9 & 40.9 & 3,676 \\
\hline op2 & 5'-TACACCTTTGTTTCCGTGGC-3' & 20 & 58.4 & 50.0 & \\
\hline op3 & 5'-AGGTAGCGGTCAAGAAGAAAT-3' & 21 & 55.4 & 42.9 & 6,965 \\
\hline op4 & 5'-GAACCGCAAAGAACTGGG-3' & 18 & 56.0 & 55.6 & \\
\hline op5 & 5'-AATATTATTGACACTCCCGGAC-3' & 22 & 55.5 & 40.9 & 2,661 \\
\hline op6 & 5'-АCTCTACCAGTAACAACAGTTCCTC-3' & 25 & 56.1 & 44.0 & \\
\hline op7 & 5'-CACATTTTATTAGCGCGCC-3' & 19 & 57.2 & 47.4 & 1,519 \\
\hline op8 & 5'-AAAACCTAACGCAATCATGG-3' & 20 & 55.5 & 40.0 & \\
\hline
\end{tabular}

RpoB、RpoC、Rps12、Rps7、FusA、TUF及其6个 基因间区 $r p l L-r p o B 、 r p o B-r p o C 、 r p o C-r p s 12 、 r p s 12-$ rps7、rps7-fusA、fusA-tuf核苷酸序列对PaWB-sdyz、 PaWB-fjfz、LY-fjya1、OY-M、AYWB、PAa、SLY、 $\mathrm{AT}$ 等 8 个植原体和 1 个非固醇甾原体 PG-8A进行 MLSA分析。

\subsection{5 tuf基因上游序列启动子活性验证}

用引物对 TPf/TPr (于少帅等, 2016b)扩增CWBhnsy1、LY-fjya1植原体株系tuf上游129-130 bp序列, 用引物对 TP4f/TPr扩增PaWB-fjfz上游130 bp序列。 将各PCR产物和pSUPV4质粒用Hind III和BamH I双 酶切后用T4 DNA连接酶连接, 连接产物通过热激 法转化大肠杆菌感受态细胞DH5 $\alpha$, 涂布于含有卡 那霉素(Kan)(Kan浓度分别为30和50 mg/L)的LB平 板上, $37^{\circ} \mathrm{C}$ 培养 $24 \mathrm{~h}$ 后观察。通过双酶切和DNA测序 验证转化子中是否存在目的片段, 引物信息见表2。

\section{2 结果}

\section{1 植原体tuf与其上游基因序列扩增}

通过引物op1/op2、op3/op4、op5/op6、op7/op8 对PaWB-sdyz、PaWB-fjfz、LY-fjya1植原体株系tuf 基因及其上游 6 个基因进行PCR扩增测序。本研究所 得核苷酸序列已上传至GenBank数据库, 3 个植原体 株系PaWB-sdyz、PaWB-fjfz和LY-fjya1的rplL-tuf核 苷酸序列的 GenBank 序列号分别为 MG744258、 MG744259和MG744260。对所得植原体序列的持家 基因及其编码的氨基酸序列和基因间区序列进行 分析。PaWB-sdyz、PaWB-fjfz、LY-fjya1植原体株 系及OY-M、AYWB、PAa、SLY、AT等8个植原体 株系和 1 个非固醇甾原体株PG-8A的7个基因和其对 应的氨基酸序列及其 6 个基因间区的核苷酸序列信 息如表3所示。tuf基因上游6个基因分别为 $r p l L$ 、 $r p o B 、 r p o C 、 r p s 12 、 r p s 7 、 f u s A, 7$ 个基因的结构顺 序均为 5'-rplL-rpoB-rpoC-rps12-rps7-fusA-tuf-3'。 将各植原体株系及非固醇甾原体PG-8A的此段基因 结构进行比较分析, 表明植原体间及植原体与非固 醇甾原体间基因结构存在一定的相似性和多样性。

\section{2 植原体及非固醇甾原体tuf 与其上游基因结构} 分析

对 $t u f$ 等 7 个基因及基因间区序列分析发现, PaWB-sdyz、PaWB-fjfz、LY-fjya1、OY-M、AYWB、 PAa、SLY植原体fusA-tuf基因间区序列长在118-130 $\mathrm{bp}$ 之间, 有明显的启动子结构特征; 非固醇甾原体 PG-8A的fusA-tuf基因间区序列长86 bp, 有明显的 启动子结构; AT植原体fusA-tuf基因间区长 $65 \mathrm{bp}$, 未 检测到完整启动子结构。PaWB-sdyz、PaWB-fjfz、

LY-fjya1、OY-M、PAa、SLY、AT植原体rps7-fusA 基因间区长27-35 bp, 基因间区未预测到完整启动 子结构; AYWB株系rps7、fusA为重叠基因; PG-8A 株系rps7-fusA基因间区长 $17 \mathrm{bp}$, 亦未检测到完整的 启动子结构。PaWB-sdyz、PaWB-fjfz、LY-fjya1、 OY-M植原体株系rps12-rps7基因间区序列长为88 $\mathrm{bp}$, 预测有完整的启动子结构; AYWB、PAa、SLY、 AT株系rps12-rps7基因间区长51-70 bp，未预测到 完整启动子结构; PG-8A株系rps12-rps7基因间区长 $66 \mathrm{bp}$ ，未预测到完整的启动子结构。PaWB- sdyz、 PaWB-fjfz、LY-fjya1、OY-M、PAa、SLY、AT植原 体株系rpoC-rps12基因间区序列长20-27 bp，未预 测到完整的启动子结构; 植原体AT株系rpoC-rps12 基因间区序列长193 bp, PG-8A株系rpoC-rps12基 因间区长 $260 \mathrm{bp}$, 有完整的启动子结构。 PaWB-sdyz、PaWB-fjfz、LY-fjya1、OY-M、PAa、 
表2 tuf基因启动子扩增引物

Table 2 Primers used to amplify tuf gene promoters

\begin{tabular}{|c|c|c|c|c|}
\hline 引物 Primer & 引物序列 Primer sequences (5’-3’) & 引物大小 Primer size (bp) & $\mathrm{T}_{\mathrm{m}}\left({ }^{\circ} \mathrm{C}\right)$ & 引入酶切位点 Restriction site added \\
\hline TPf & cccaagcttACAACCTTACACTAAAAAAC & 29 & 45 & Hind III \\
\hline TP4f & cccaagcttACAACСТTAAACTAAAAAAC & 29 & 45 & Hind III \\
\hline TPr & cgcggatcCATTTTTCAAAGGCCTC & 25 & 45 & BamH I \\
\hline
\end{tabular}

表3 植原体扩增、编码区、非编码区序列和编码氨基酸序列及参照株系对应片段长度

Table 3 Length of sequence amplified, coding region, non-coding region and amino acid sequence of phytoplasmas as well as reference strains

\begin{tabular}{llllll}
\hline $\begin{array}{l}\text { 株系 } \\
\text { Strain }\end{array}$ & $\begin{array}{l}\text { 级别 } \\
\text { Group }\end{array}$ & $\begin{array}{l}\text { 扩增序列长度 } \\
\text { Sequence amplified } \\
\text { length (bp) }\end{array}$ & $\begin{array}{l}\text { 编码区长度 } \\
\text { Coding region } \\
\text { length (bp) }\end{array}$ & $\begin{array}{l}\text { 非编码区长度 } \\
\text { Non-coding region } \\
\text { length (bp) }\end{array}$ & $\begin{array}{l}\text { 氨基酸长度/个 } \\
\text { Amino acid sequence } \\
\text { length }\end{array}$ \\
\hline PaWB-sdyz & 16SrI-D & 12,746 & 12,307 & 439 & 4,096 \\
PaWB-fjfz & 16SrI-D & 12,745 & 12,307 & 438 & 4,096 \\
LY-fjya1 & 16 SrI-B & 12,748 & 12,307 & 441 & 4,096 \\
OY-M & 16 SrI-B & 12,745 & 12,307 & 438 & 4,096 \\
AYWB & 16 SrI-A & 12,735 & 12,343 & 392 & 4,107 \\
PAa & $16 S r X I I$ & 12,611 & 12,211 & 400 & 4,063 \\
SLY & $16 S r X I I$ & 12,611 & 12,211 & 400 & 4,063 \\
AT & $16 S r X$ & 12,835 & 12,301 & 534 & 4,093 \\
PG-8A & & 4,587 & 4,158 & 429 & 1,382 \\
\hline
\end{tabular}

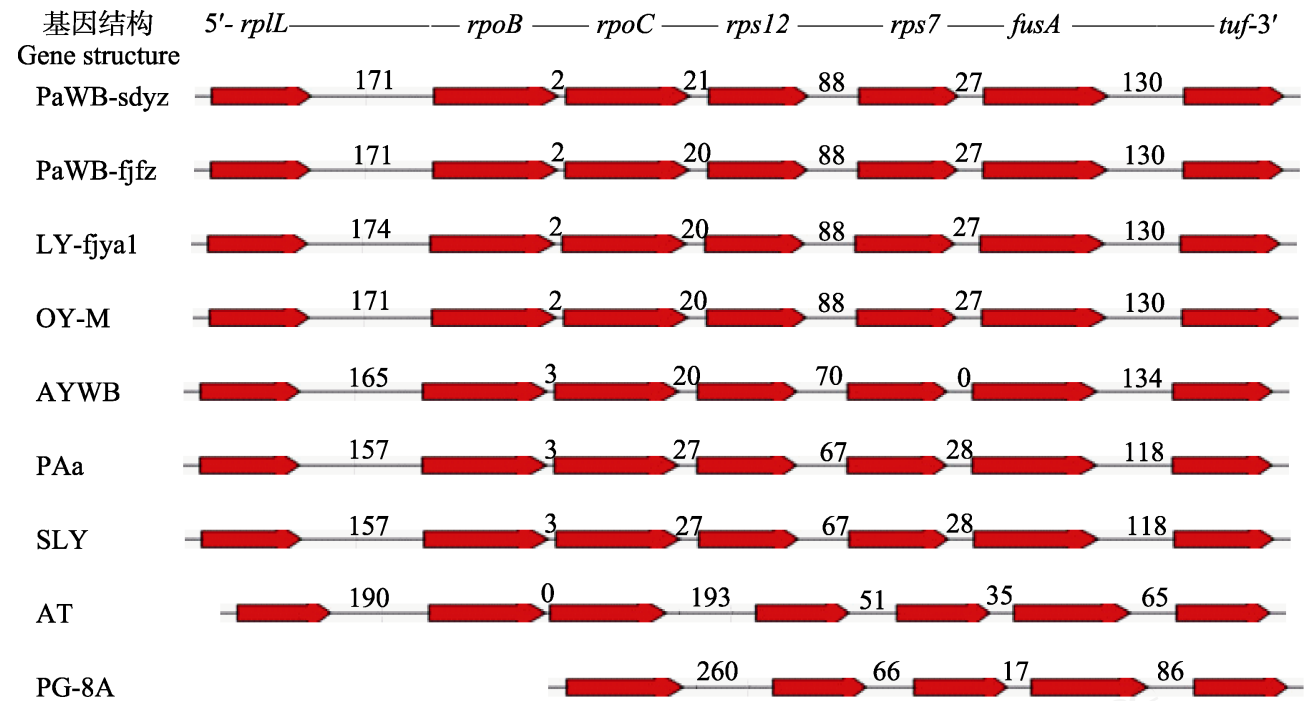

图1 不同植原体tuf基因及其上游基因结构示意图。图中样品代号见正文“1.1”和“1.2.3”; 数字代表基因间区序列长度(bp)。 Fig. 1 Gene structure diagram of tuf gene and its upstream genes from different phytoplasmas. The codes of samples in the figure were shown in "1.1" and “1.2.3”. Numbers represented the length of intergenic sequences (bp).

SLY植原体 $r p o B-r p o C$ 基因间区序列长2-3 bp, 无完 整启动子结构; $\mathrm{AT}$ 株系 $r p o \mathrm{~B} 、 r p o C$ 基因为重叠基因。 PaWB-sdyz、PaWB-fjfz、LY-fjya1、OY-M、PAa、 SLY、AT植原体 rplL-rpoB 基因间区序列长157$190 \mathrm{bp}$, 预测具有完整的启动子保守区域。与植原 体各株系有所差异的是, 非固醇甾原体PG-8A株系 $r p s 12$ 基因上游的 2 个基因编码的蛋白产物为假设蛋 白, 植原体各株系的 rplL-rpoB和 rpoB-rpoC这两个 基因间区序列未与PG-8A株系进行比较分析(图1)。

2.3 植原体和非固醇甾原体相关基因启动子保守 结构特征

已有研究发现：16SrI组植原体fusA-tuf基因间区 
序列存在 4 种变异类型, 并预测有完整的启动子结 构(于少帅等, 2016b), 相关植原体基因启动子保守 区域特征序列如表4所示。根据研究中所涉及的 52 个可能的植原体启动子保守区域序列特征，对部分 植原体部分基因启动子保守区域不同位置核苷酸 种类及出现频率进行了统计分析(表5), 根据保守区 域不同位置核苷酸种类及出现频率推测出植原体 启动子保守区域可能的序列模式为： -35 区 $\mathrm{T}_{90} \mathrm{~T}_{100} \mathrm{G}_{92} \mathrm{~T}_{75} \mathrm{G}_{67} \mathrm{~A}_{85},-10$ 区 $\mathrm{T}_{90} \mathrm{~A}_{96} \mathrm{~T}_{92} \mathrm{~A}_{98} \mathrm{~T}_{73} \mathrm{~T}_{90}$ 。

\subsection{MLSA分析}

基于 8 个植原体株系 rplL-tuf基因序列进行 MLSA分析, 8个植原体株系rplL-tuf核苷酸编码区整 合序列总长为12,301-12,343 bp; 8 个植原体株系 rplL-tuf核苷酸编码区整合序列相似性为 72.7-
99.9\%; 非固醇甾原体PG-8A株系与8个植原体株系 rplL-tuf核苷酸编码区整合序列相似性为 70.971.4\%。基于编码区整合序列的进化树表明: $16 \mathrm{SrI}-\mathrm{D}$ 亚组PaWB-sdyz、PaWB-fjfz株系以95\%的支持率聚 于一支, 16SrI-B亚组的洋葱黄化植原体OY-M、莴亘 黄化植原体LY-fjya1和16SrI-A亚组的AYWB分别单 独聚于一支，支持率均为 $99 \%, 16 \mathrm{SrXII}$ 组的两个株 系PAa、SLY以 $100 \%$ 的支持率单独聚于一支(表6、 图2)。

基于 8 个植原体株系rplL-tuf基因间区非编码序 列进行MLSA分析, 8 个植原体株系rplL-tuf核苷酸非 编码区整合序列总长为392-534 bp; 8株植原体株系 rplL-tuf核苷酸非编码区整合序列相似性为50.7100\%; PG-8A株系与8个植原体株系rplL-tuf核苷酸

表4 植原体rplL-tuf基因间区序列相关基因启动子保守区域特征

Table 4 Characteristics of conserved region of relevant gene promoter of phytoplasma rplL-tuf intergenic sequences

\begin{tabular}{|c|c|c|c|c|c|c|c|c|}
\hline \multirow{2}{*}{$\begin{array}{l}\text { 株系 } \\
\text { Strains }\end{array}$} & \multicolumn{2}{|l|}{ rplL-rроB } & \multicolumn{2}{|c|}{ rpoC-rps12 } & \multicolumn{2}{|l|}{ rps12-rps7 } & \multicolumn{2}{|l|}{ fusA-tuf } \\
\hline & -35 & -10 & -35 & -10 & -35 & -10 & -35 & -10 \\
\hline PaWB-sdyz & TTGCAT & TATACC & - & - & ATAAAA & AAAAAT & TTGTGA & TATATT \\
\hline PaWB-fjfz & TTGCAT & TATACC & - & - & ATAAAA & AAAAAT & TTGTGA & TATATT \\
\hline LY-fjya1 & TTGCAT & TATACC & - & - & ATAAAA & AAAAAT & TTGTAA & TATATT \\
\hline OY-M & TTGAAT & TATAAC & - & - & ATAAAA & AAAAAT & TTGTGA & TATATT \\
\hline AYWB & TTGCAT & TATACC & - & - & - & - & TTGTGA & TATTAT \\
\hline PAa & TTGTAT & TTTAAT & - & - & - & - & TTGATA & TATATT \\
\hline SLY & TTGTAT & TTTAAT & - & - & - & - & TTGATA & TATATT \\
\hline AT & ATGATA & AATAAT & TTGACT & TATAAT & - & - & - & - \\
\hline PG-8A & - & - & TTGACA & TATAAT & - & - & ATGATA & TATTGT \\
\hline
\end{tabular}

31个植原体株系fusA-tuf基因间区启动子保守区域特征序列与PaWB-sdyz株系一致，分别为泡桐丛枝植原体株系PaWB-jsnj、-jssz1、-jssz2、 -bjhr、-hnzz、-hnpy、-hnly、-hncs、-ahhf、-jxnc、-hbbd、-lndl1、-lndl2、-sxty1、-sxty2、-sxxa1、-sxxa2、-sxwn (18个株系); 长春花绿变植 原体株系PeV-hnhk (1个株系); 苦楝从枝植原体株系CWB-hnsy1、-hnsy2、-fjfz1、-fjfz2、-fjfz3、-fjya、-jsnj、-hncs、-jxnc、-gdgz (10个株系); 桑萎缩植原体株系MD-ahhf和-zjca (2个株系)。莴芭黄化植原体株系LY-fjya2株系fusA-tuf基因间区启动子保守区域特征序列与LY-fjya1株系一 致(于少帅等, 2016b)。

Characteristic sequences of conserved regions of fusA-tuf intergenic sequence of 31 phytoplasma strains are as same as PaWB-sdyz, including PaWB-jsnj, -jssz1, -jssz2, -bjhr, -hnzz, -hnpy, -hnly, -hncs, -ahhf, -jxnc, -hbbd, -lndl1, -lndl2, -sxty1, -sxty2, -sxxa1, -sxxa2 and -sxwn (18 strains); PeV-hnhk (1 strains); CWB-hnsy1, -hnsy2, -fjfz1, -fjfz2, -fjfz3, -fjya, -jsnj, -hncs, -jxnc, -gdgz (10 strains); MD-ahhf and -zjca (2 strains). Characteristic sequences of conserved regions of fusA-tuf intergenic sequence of LY-fjya2 strain are as same as LY-fjya1 strain (Yu et al, 2016b).

表5 部分植原体基因启动子保守区域不同位置核苷酸种类及出现频率

Table 5 Nucleotide and its frequency in different sites of conserved regions of some phytoplasma gene promoters

\begin{tabular}{|c|c|c|c|c|c|c|c|c|c|c|c|c|}
\hline \multirow{2}{*}{$\begin{array}{l}\text { 保守区域 Conserved region } \\
\text { 序列特征 } \\
\text { Sequence characteristic }\end{array}$} & \multicolumn{6}{|c|}{-35区 -35 region } & \multicolumn{6}{|c|}{-10区 -10 region } \\
\hline & $\mathrm{A}(10)$ & $\mathrm{A}(0)$ & $\mathrm{A}(8)$ & $\mathrm{A}(17)$ & $\mathrm{A}(25)$ & $\mathbf{A}(85)$ & $\mathrm{A}(10)$ & $A(96)$ & $\mathrm{A}(8)$ & $\mathrm{A}(98)$ & $\mathrm{A}(19)$ & $\mathrm{A}(0)$ \\
\hline \multirow[t]{3}{*}{ Sequence characteristic } & $\mathbf{T}(90)$ & $T(100)$ & $\mathrm{T}(0)$ & $\mathbf{T}(75)$ & $\mathrm{T}(6)$ & $\mathrm{T}(15)$ & $\mathbf{T}(90)$ & $\mathrm{T}(4)$ & $\mathrm{T}(92)$ & $\mathrm{T}(2)$ & $\mathbf{T}(73)$ & $\mathrm{T}(\mathbf{9 0})$ \\
\hline & $\mathrm{G}(0)$ & $\mathrm{G}(0)$ & G(92) & $\mathrm{G}(0)$ & G(67) & $\mathrm{G}(0)$ & $\mathrm{G}(0)$ & $\mathrm{G}(0)$ & $\mathrm{G}(0)$ & $\mathrm{G}(0)$ & $\mathrm{G}(0)$ & $\mathrm{G}(0)$ \\
\hline & $\mathrm{C}(0)$ & $\mathrm{C}(0)$ & $\mathrm{C}(0)$ & C(8) & $\mathrm{C}(2)$ & $\mathrm{C}(0)$ & $\mathrm{C}(0)$ & $\mathrm{C}(0)$ & $\mathrm{C}(0)$ & $\mathrm{C}(0)$ & C(8) & $\mathrm{C}(10)$ \\
\hline
\end{tabular}

-35 区、 -10 区序列不同位置出现的核苷酸频率在碱基后括号中标出(\%); 统计的植原体基因启动子个数 $n=52$ 。

Frequencies of nucleotide in different sites of -35 and -10 regions are listed in parentheses (\%). Statistic numbers of phytoplasma gene promoters are $\mathrm{n}=52$. 
表6 MLSA分析rplL-tuf核苷酸序列编码区同源比对

Table 6 Homology matrix of rplL-tuf nucleotide sequence coding region in MLSA analysis

\begin{tabular}{|c|c|c|c|c|c|c|c|c|c|}
\hline 株系 Strain & PaWB-sdyz & PaWB-fjfz & LY-fjya1 & OY-M & AYWB & РАa & SLY & $\mathrm{AT}$ & PG-8A \\
\hline PaWB-sdyz & 100 & & & & & & & & \\
\hline PaWB-fjfz & 99.7 & 100 & & & & & & & \\
\hline LY-fjya1 & 99.3 & 99.3 & 100 & & & & & & \\
\hline OY-M & 99.6 & 99.6 & 99.4 & 100 & & & & & \\
\hline AYWB & 95.9 & 95.9 & 95.9 & 96.0 & 100 & & & & \\
\hline PAa & 81.1 & 81.1 & 81.2 & 81.1 & 81.1 & 100 & & & \\
\hline SLY & 81.1 & 81.1 & 81.2 & 81.1 & 81.1 & 99.9 & 100 & & \\
\hline AT & 72.7 & 72.8 & 72.9 & 72.8 & 73.3 & 73.6 & 73.6 & 100 & \\
\hline PG-8A & 71.2 & 71.3 & 71.4 & 71.3 & 71.4 & 71.3 & 71.3 & 70.9 & 100 \\
\hline
\end{tabular}

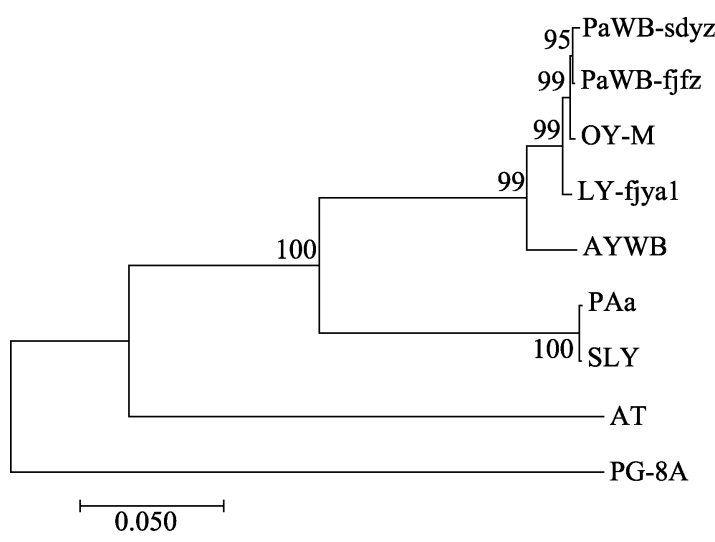

图2 基于rplL-tuf核苷酸序列编码区整合序列构建的植原 体系统发育树。图中代号见“1.1”和“1.2.3”。

Fig. 2 Phylogenetic tree of the phytoplasma strains reconstructed based on the concatenated gene sequences data set of rplL-tuf nucleotide sequence coding region. The codes in the figure were shown in "1.1" and "1.2.3".

非编码区整合序列相似性为40.2-44.8\%。基于植原 体rplL-tuf基因间区非编码序列的多位点序列分析 表明：16SrI-D亚组PaWB-fjfz植原体株系以79\%的
支持率单独聚于一支，16Sr-D亚组PaWB-sdyz、 16SrI-B亚组OY-M、LY-fjya1共3个株系以 $86 \%$ 的支 持率单独聚于一个大的进化分支，与编码区的分析 结果有出入; 16SrXII组的两个株系PAa、SLY以99\% 的支持率单独聚于一支，与编码区的分析结果一致 (表7, 图3)。

基于 8 个植原体株系rplL-tuf基因编码的氨基酸 序列RplL、RpoB、RpoC、Rps12、Rps7、FusA、 T U F 进行 M L S A 分析发现, 8 个植原体株系 RplL-TUF氨基酸整合序列总长为4,063-4,107个氨 基酸，相似性为69.0-99.8\%; PG-8A株系与8株植原 体株系 RplL-T UF氨基酸整合序列相似性为 70.4-75.1\%。基于8个植原体株系RplL-TUF氨基酸 整合序列构建的系统发育树表明：16SrI-D亚组 PaWB-sdyz、PaWB-fjfz株系以58\%的支持率单独聚 于一进化支，16SrI-B亚组的OY-M、LY-fjya1和 16SrI-A亚组的AYWB分别单独聚于一支, 支持率分 别为 $96 \% 、 80 \% 、 100 \%, 16 \operatorname{SrXII}$ 组的两个株系PAa、

表7 MLSA分析rplL-tuf核苷酸序列非编码区同源比对

Table 7 Homology matrix of rplL-tuf nucleotide sequence non-coding region in MLSA analysis

\begin{tabular}{|c|c|c|c|c|c|c|c|c|c|}
\hline 株系 Strain & PaWB-sdyz & PaWB-fjfz & LY-fjya1 & OY-M & AYWB & РАa & SLY & AT & PG-8A \\
\hline PaWB-sdyz & 100 & & & & & & & & \\
\hline PaWB-fjfz & 99.3 & 100 & & & & & & & \\
\hline LY-fjya1 & 99.3 & 98.6 & 100 & & & & & & \\
\hline OY-M & 99.3 & 98.6 & 98.6 & 100 & & & & & \\
\hline AYWB & 94.6 & 94.3 & 94.1 & 94.1 & 100 & & & & \\
\hline PAa & 71.9 & 71.8 & 71.3 & 72.3 & 73.2 & 100 & & & \\
\hline SLY & 71.9 & 71.8 & 71.3 & 72.3 & 73.2 & 100 & 100 & & \\
\hline AT & 50.7 & 51.3 & 51.4 & 51.3 & 51.1 & 51.4 & 51.4 & 100 & \\
\hline PG-8A & 41.8 & 41.3 & 41.5 & 41.8 & 41.3 & 40.2 & 40.2 & 44.8 & 100 \\
\hline
\end{tabular}




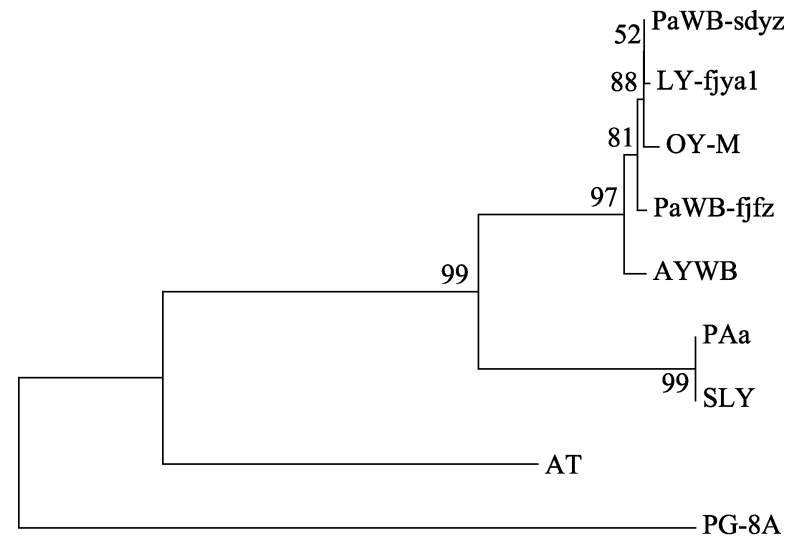

$\longmapsto 0.10$

图3 基于rplL-tuf核苷酸序列非编码区整合序列构建的植 原体系统发育树。图中株系代号见正文“1.1”和“1.2.3”。

Fig. 3 Phylogenetic tree of the phytoplasma strains reconstructed based on the concatenated gene sequences data set of rplL-tuf nucleotide sequence non-coding region. The codes in the figure are shown in "1.1" and "1.2.3".

SLY以 $100 \%$ 的支持率单独聚于一支(表8，图4)。

\section{$2.5165 r I$ 组植原体tuf基因上游序列启动子活性} 验证

研究发现16SrI组植原体fusA-tuf基因间区存在4 种启动子变异类型, 实验验证表明PaWB-sdyz株系 代表的变异类型TP1 (GenBank序列号: KU563013) 具有启动子活性(于少帅等, 2016b)。植原体株系 CWB-hnsy1、LY-fjya1和PaWB-fjfz分别代表16SrI组 植原体fusA-tuf基因间区序列变异类型TP2、TP3和 TP4 (于少帅等, 2016b)。用引物对 TPf/TPr获取 CWB-hnsy1、LY-fjya1植原体株系fusA-tuf基因间区 序列129 bp、130 bp; 用引物对TP4f/TPr获取PaWBfjfz株系fusA-tuf基因间区序列130 bp。利用启动子探
针载体pSUPV4构建的融合表达体系检测表明: 在 含 30 和 $50 \mathrm{mg} / \mathrm{L} \mathrm{Kan}$ 的LB平板上培养 $24 \mathrm{~h}$ 后, 利用 植原体CWB-hnsy1、LY-fjya1和PaWB-fjfz株系fusAtuf基因间区序列构建的融合表达体系在 LB平板上 均有明显的转化子产生, 挑取单菌落测序发现转化 子中含有目的片段。因而判断3株植原体株系fusAtuf基因间区具有启动子活性。

\section{3 讨论}

本研究扩增获取了我国3个植原体株系tuf基因 上游近13 kb的序列, 不同植原体株系tuf与其上游 6 个基因的结构顺序一致(Oshima et al, 2004; Bai et al, 2006; Kube et al, 2008; Tran-Nguyen et al, 2008; Andersen et al, 2013)。PaWB-sdyz、PaWB-fjfz、 LY-fjya1、OY-M、AYWB、PAa、SLY等7个植原体 株系fusA-tuf基因间区长为118-134 bp, 预测有完整 的启动子结构, 启动子探针检测表明PaWB-sdyz (于少帅等, 2016b)、PaWB-fjfz、LY-fjya1、CWBhnsy1等16SrI组植原体fusA-tuf基因间区4种序列变 异类型均具有启动子活性。由此推测, tuf基因在 $16 S r I$ 组植原体中可以单独启动表达, 而不必与上 游rps12、rps7、fusA基因一起构成操纵子。然而, Miyata等的研究推测表明洋苾黄化植原体OY株系 tuf基因是与上游的 rps 12、rps7 和fus $A$ 等 3 个基因一起 构成了植原体的str操纵子(Miyata et al, 2002b), 但 这一推断并未通过实验进行验证。因而我们推测由 于植原体基因结构及其表达调控本身的复杂性 (Salehi et al, 2017; Liu et al, 2017)，不同植原体tuf基 因的转录表达调控存在不同的方式。在某些情况下,

表8 MLSA分析RplL-TUF蛋白氨基酸序列同源比对

Table 8 Homology matrix of RplL-TUF amino acid sequence in MLSA analysis

\begin{tabular}{|c|c|c|c|c|c|c|c|c|c|}
\hline 株系 Strain & PaWB-sdyz & PaWB-fjfz & LY-fjya1 & OY-M & AYWB & РАа & SLY & AT & PG-8A \\
\hline PaWB-sdyz & 100 & & & & & & & & \\
\hline PaWB-fjfz & 99.6 & 100 & & & & & & & \\
\hline LY-fjya1 & 98.9 & 99.0 & 100 & & & & & & \\
\hline OY-M & 99.4 & 99.5 & 99.2 & 100 & & & & & \\
\hline AYWB & 97.8 & 97.9 & 98.0 & 98.0 & 100 & & & & \\
\hline РAa & 83.8 & 83.9 & 84.1 & 83.9 & 83.7 & 100 & & & \\
\hline SLY & 83.8 & 83.9 & 84.1 & 83.9 & 83.7 & 99.8 & 100 & & \\
\hline AT & 69.8 & 69.9 & 70.0 & 69.9 & 69.8 & 69.0 & 69.0 & 100 & \\
\hline PG-8A & 74.9 & 75.1 & 74.9 & 75.0 & 74.8 & 74.1 & 73.9 & 70.4 & 100 \\
\hline
\end{tabular}




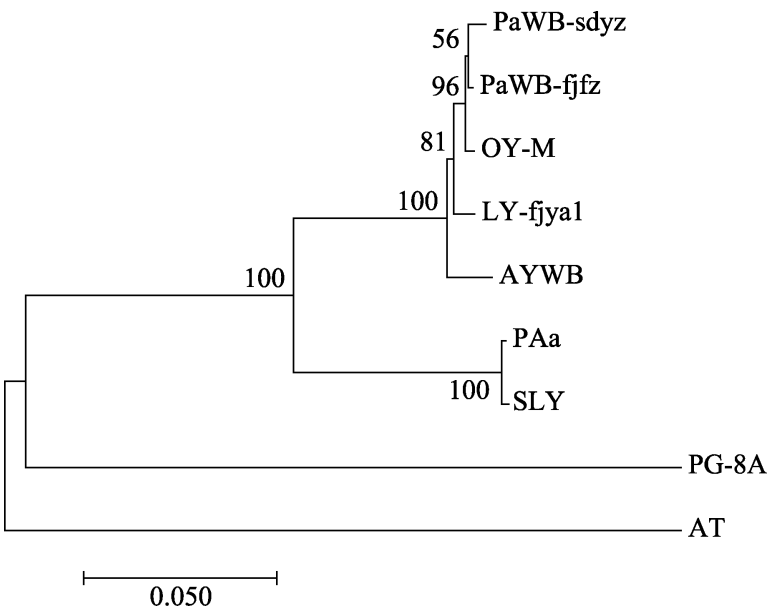

图4 基于RplL-TUF氨基酸整合序列构建的植原体系统发 育树。图中代号见正文“1.1”和“1.2.3”。

Fig. 4 Phylogenetic tree of the phytoplasma strains reconstructed based on the concatenated sequences data set of RplL-TUF amino acid sequence. The codes in the figure are shown in "1.1" and "1.2.3”.

植原体tuf基因可以不与 rps12、rps7、fusA等上游基 因一起转录, 而是单独转录表达。关于植原体内 $t u f$ 基因及其与上游 $r p l L 、 r p o B 、 r p o C 、 r p s 12 、 r p s 7 、$ fus $A$ 基因的具体转录表达的详细调控过程, 有待于 相关研究进一步验证。

研究表明不同植原体质粒上和染色体DNA上 不同基因的启动子在-35和-10位的特征序列核苷 酸都有差异(Ishii et al, 2009)。在对PaWB-sdyz、 PaWB-fjfz、LY-fjya1、OY-M、AYWB、PAa、SLY、 AT等 8 个植原体株系rplL-tuf基因结构分析过程中, 对具有完整启动子结构的启动子保守区域的-10区 和-35区的特征序列进行了分析, 同时结合之前已 测序的我国不同地区、不同寄主的16SrI组植原体株 系的fusA-tuf基因间区序列进行统计分析, 推测植原 体基因启动子保守区域可能的序列模式为：-35区 $\mathrm{T}_{90} \mathrm{~T}_{100} \mathrm{G}_{92} \mathrm{~T}_{75} \mathrm{G}_{67} \mathrm{~A}_{85},-10$ 区 $\mathrm{T}_{90} \mathrm{~A}_{96} \mathrm{~T}_{92} \mathrm{~A}_{98} \mathrm{~T}_{73} \mathrm{~T}_{90}$ 。已知 -35 区序列的功能是为RNA聚合酶的识别提供信号, 为识别域; 而 -10 区序列允许复合体由闭合转变为 开放, 为解旋域。由于破坏 $\mathrm{A}-\mathrm{T}$ 碱基对使双链分离需 要的能量小于 G-C碱基对, 故-10区的共有序列对 主要为AT (李明刚, 2004)。Ishii等(2009)对洋葱黄化 植原体野生株系OY-M和其变异株系非虫传株系 OY-NIM质粒ORF1、ORF2基因启动子保守区域的 -10 区和-35区的特异序列进行了预测分析, 并与 OY-M株系16S rRNA、Amp基因启动子保守区域的 -10 区和 -35 区的特异序列对比分析, 各启动子保守
区域特征序列为OY-M ORF1：-35区TTCAAT, -10 区 TATTGA; OY-M ORF2: -35区 TTTATA，-10区 TTAATT; OY-NIM ORF1: -35区CTTAAT, -10区 TATTGA; OY-M 16S rDNA: -35区TTGAAA, -10区 TATAAT; OY-M Amp: -35 区 TTGTTA, -10 区 TATAAT。由此发现植原体不同株系、不同功能基 因启动子保守区域-35区和-10区的特征序列有所 差异。但是, 由于统计分析研究中所涉及的已验证 确切功能的启动子数量有限, 难以较为系统、全面 地揭示植原体启动子保守区域的序列特征，因此， 本研究所得到的植原体启动子保守区域可能的序 列模式在植原体不同基因启动子中是否具有普遍 性和代表性，还有待于后期通过生物信息学方法对 大量植原体不同基因或操纵子启动子保守区域进 行预测和功能验证分析。

充分的研究已揭示大肠杆菌基因启动子-35 区、 -10 区序列模式为： -35 区 $\mathrm{T}_{85} \mathrm{~T}_{83} \mathrm{G}_{81} \mathrm{~A}_{61} \mathrm{C}_{69} \mathrm{~A}_{52}$, -10 区 $\mathrm{T}_{89} \mathrm{~A}_{89} \mathrm{~T}_{50} \mathrm{~A}_{65} \mathrm{~A}_{100}$ (朱玉贤和李毅, 2002)。由此 可见植原体与大肠杆菌的启动子保守区域结构保 守序列碱基既有共同的特征，也存在一定的差异。 模式序列的-35区存在两个碱基差异，碱基 $\mathrm{T}$ 颠换为 $\mathrm{A}$, 碱基 $\mathrm{G}$ 颠换为 $\mathrm{C} ;-10$ 区有一个碱基的差异，碱基 $\mathrm{T}$ 颠换为 $\mathrm{A}$ 。由于原核细胞中只有一种RNA聚合酶 参与转录过程, 细菌基因的转录起始频率直接与启 动子序列的结构差异有关; 可能这种差异反映到某 个启动子序列与共有序列的同源程度方面，如果同 源程度低则起始频率会低(杨业华, 2008)。植原体不 同株系不同功能基因启动子保守区域序列及其与 大肠杆菌启动子保守区域序列模式、非固醇甾原体 部分相同基因启动子保守区域序列存在的碱基差 异，必定影响不同细菌、不同基因在细菌不同生活 阶段、不同环境下的表达及其表达效率，从而达到 适应各自不同生态环境的目的(朱玉贤和李毅, 2002; Du et al, 2014; Zhang et al, 2014)。

基于 $r p l L$-tuf共7个基因、其对应的氨基酸序列 RplL、RpoB、RpoC、Rps12、Rps7、FusA、TUF 及其6个基因间区序列 $r p l L-r p o B 、 r p o B-r p o C 、 r p o C$ -rps12、rps12-rps7、rps7-fusA、fusA-tuf的MLSA分 析可准确、清晰地将PaWB-sdyz等8个植原体株系和 1个非固醇甾原体株系区分。根据序列相似性、系 统发育树拓扑结构和支持率可知，与rplL-tuf核苷酸 编码区相比, 不同植原体株系rplL-tuf核苷酸非编码 
区变异水平较高, 相似性范围幅度较大。不同植原 体株系rplL-tuf核苷酸编码区和RplL-TUF氨基酸序 列相似性略有差异, RplL-TUF氨基酸序列变异水平 相对较高。除了基于RplL-TUF氨基酸序列构建的系 统发育树的AT株系外, 不同类型序列构建的系统 发育树遗传距离有所差异, 拓扑结构基本一致。已 有研究证据显示, 以山东充州株系PaWB-sdyz为代 表的泡桐丛枝植原体优势株系与福建福州株系 PaWB-fjfz间有显著的遗传分化现象, 后者引起的 症状、16SrDNA、许多持家基因及质粒DNA序列都 发生了明显的变异(任争光, 2014; 王圣洁, 2017)。 基于 rplL-tuf编码区序列 MLSA 山东充州株系 PaWB-sdyz为代表的泡桐从枝植原体优势株系与福 建福州株系PaWB-fjfz的亲缘关系仍最相近, 然而 基于非编码序列的MLSA 福建福州株系PaWB-fjfz 却以较高的支持率聚为单独的一支, 相反 PaWB-sdyz株系却与LY-fjya1、OY-M聚为一支。因 而推断仅用有限非编码序列可能难以准确反映植 原体株系间的系统进化和亲缘关系远近; 非编码区 的更高的变异频率, 更可能与地理、生态隔离导致 的中性突变有关。所以根据目前的研究结果来看, 基于rplL-tuf基因簇核苷酸编码区构建的系统发育 树可将不同组别、不同亚组的植原体株系以较高的 支持率清晰地区分, 并且可将同为 $165 r I-B$ 亚组不 同寄主的植原体株系清晰地分开。由此可知, 基于 $r p l L-t u f$ 基因簇核苷酸编码区序列的MLSA分析可在 16S rRNA、 $r p$ 等基因分类鉴定的基础上，作为一种 可能的手段用于不同地区不同寄主植原体株系遗 传分化研究中, 以揭示植原体更为丰富、细淢的遗 传变异规律和系统进化关系, 为植原体鉴定、分类 及其相关病害的流行监控、防治管理等提供一种 较为有效的参考手段。

\section{参考文献}

Andersen MT, Liefting LW, Havukkala I, Beever RE (2013) Comparison of the complete genome sequence of two closely related isolates of 'Candidatus phytoplasma australiense’ reveals genome plasticity. BMC Genomics, 14, 529.

Bai XD, Zhang JH, Ewing A, Miller SA, Radek AJ, Shevchenko DV, Tsukerman K, Walunas T, Lapidus A, Campbell JW, Hogenhout SA (2006) Living with genome instability: The adaptation of phytoplasma to diverse environments of their insect and plant hosts. Journal of Bacteriology, 188, 3682-3696.
Du HT, Zhu HY, Wang JM, Zhao W, Tao XL, Ba CF, Tian YM, Su YH (2014) Single-nucleotide polymorphisms and activity analysis of the promoter and enhancer of the pig lactase gene. Gene, 545, 56-60.

Felsenstein J (1985) Confidence limits on phylogenies: An approach using the bootstrap. Evolution, 39, 783-791.

Ishii Y, Kakizawa S, Hoshi A, Maejima K, Kagiwada S, Yamaji Y, Oshima K, Namba S (2009) In the non-insecttransmissible line of onion yellows phytoplasma (OY-NIM), the plasmid-encoded transmembrane protein ORF3 lacks the major promoter region. Microbiology, 155, 2058-2067.

Kube M, Schneider B, Kuhl H, Dandekar T, Heitmann K, Migdoll AM, Reinhardt R, Seemüller E (2008) The linear chromosome of the plant-pathogenic mycoplasma 'Candidatus phytoplasma mali’. BMC Genomics, 9, 306.

Kumar S, Stecher G, Tamura K (2016) MEGA7: Molecular evolutionary genetics analysis version 7.0 for bigger datasets. Molecular Biology and Evolution, 33, 1870-1874.

Lee IM, Hammond RW, Davis RE, Gundersen DE (1993) Universal amplification and analysis of pathogen 16S rDNA for classification and identification of mycoplasma like organisms. Phytopathology, 83, 834-842.

Li MG (2004) Advanced Molecular Genetics. Science Press, Beijing. (in Chinese) [李明刚 (2004) 高级分子遗传学. 科 学出版社, 北京.]

Liu Z, Zhang L, Xue C, Fang H, Zhao J, Liu M (2017) Genome-wide identification and analysis of MAPK and MAPKK gene family in Chinese jujube (Ziziphus jujuba Mill.). BMC Genomics, 18, 855.

Miyata S, Furuki K, Oshima K, Sawayanagi T, Nishigawa H, Jung HY, Ugaki M, Namba S (2002a) Complete nucleotide sequence of the $S 10$-spc operon of phytoplasma: Gene organization and genetic code resemble those of Bacillus subtilis. DNA and Cell Biology, 21, 527-534.

Miyata S, Furuki K, Sawayanagi T, Oshima K, Kuboyama T, Tsuchizaki T, Ugaki M, Namba S (2002b) Gene arrangement and sequence of str operon of phytoplasma resemble those of Bacillus more than those of Mycoplasma. Journal of General Plant Pathology, 68, 62-67.

Oshima K, Kakizawa S, Nishigawa H, Jung HY, Wei W, Suzuki S, Arashida R, Nakata D, Miyata S, Ugaki M, Namba S (2004) Reductive evolution suggested from the complete genome sequence of a plant-pathogenic phytoplasma. Nature Genetics, 36, 27-29.

Post LE, Nomura M (1980) DNA sequences from the str operon of Escherichia coli. The Journal of Biological Chemistry, 255, 4660-4666.

Ren ZG (2014) The Qualitative and Quantitative Detection and Identification of Several Important Phytoplasmas in China. Postdoctoral Research Report, Chinese Academy of Forestry, Beijing. (in Chinese with English abstract) [任争光 (2014) 我国几种重要植原体定性和定量检测鉴定研究. 博士后研究报告, 中国林业科学研究院, 北京.] 
Salehi M, Hosseini SAE, Salehi E, Bertaccini A (2017) Genetic diversity and vector transmission of phytoplasmas associated with sesame phyllody in Iran. Folia Microbiologica, 62, 99-109.

Sanangelantoni AM, Tiboni O (1993) The chromosomal location of genes for elongation factor Tu and ribosomal protein S10 in the cyanobacterium Spirulina platensis provides clues to the ancestral organization of the str and S10 operons in prokaryotes. Journal of General Microbiology, 139, 2579-2584.

Tran-Nguyen LTT, Kube M, Schneider B, Reinhardt R, Gibb KS (2008) Comparative genome analysis of 'Candidatus phytoplasma australiense’ (subgroup tuf-Australia I; rp-A) and 'Ca. phytoplasma asteris' strains OY-M and AY-WB. Journal of Bacteriology, 190, 3979-3991.

Turner PC (translated by Liu JY, Liu WY) (2010) Molecular Biology, 3rd edn. Science Press, Beijing. (in Chinese) [特纳 (著), 刘进元, 刘文颖 (译) (2010) 分子生物学, 第3版. 科学出版社, 北京.]

Wang SJ (2017) Establishment of Molecular Detection Technology and Genetic Diversity Analysis of Plant Phytoplasma in China. PhD dissertation, Chinese Academy of Forestry, Beijing. (in Chinese with English abstract) [王圣洁 (2017) 重要林木植原体分子检测技术的研发和遗传多样性研究. 博士学位论文, 中国林业科学研究院, 北京.]

Yang YH (2008) Molecular Biology of the Gene. Higher Education Press, Beijing. (in Chinese) [杨业华 (2008) 基因的 分子生物学. 高等教育出版社, 北京.]
Yu SS, Xu QC, Lin CL, Wang SJ, Tian GZ (2016a) Genetic diversity of phytoplasmas: Research status and prospects. Biodiversity Science, 24, 205-215. (in Chinese with English abstract) [于少帅, 徐启聪, 林彩丽, 王圣洁, 田国忠 (2016a) 植原体遗传多样性研究现状与展望. 生物多样 性, 24, 205-215.]

Yu SS, Lin CL, Pan J, Ren ZG, Piao CG, Wang LF, Guo MW, Tian GZ (2016b) Comparative analysis of structure, function and genetic variation of upstream sequences adjoining tuf gene in paulownia and jujube witches'-broom phytoplasmas. Microbiology China, 43, 1060-1069. (in Chinese with English abstract) [于少帅, 林彩丽, 潘皎, 任争光, 朴春根, 汪来发, 郭民伟, 田国忠 (2016b) 泡桐从枝和麥疯病植 原体tuf基因上游序列结构、功能和遗传变异比较分析. 微 生物学通报, 43, 1060-1069.]

Zhang Y, Han Q, Li C, Li W, Fan H, Xing Q, Yan B (2014) Genetic analysis of the TBX1 gene promoter in indirect inguinal hernia. Gene, 535, 290-293.

Zhu YX, Li Y (2002) Modern Molecular Biology, 2nd edn. Higher Education Press, Beijing. (in Chinese) [朱玉贤, 李 毅 (2002) 现代分子生物学, 第2版. 高等教育出版社, 北京.]

Zurawski G, Zurawski SM (1985) Structure of the Escherichia coli S10 ribosomal protein operon. Nucleic Acids Research, 13, 4521-4526.

(责任编委: 刘杏忠 责任编辑: 时意专) 